\title{
Association between Obsessive-Compulsive Symptoms and Long-Term Cardiac Outcomes in Patients with Acute Coronary Syndrome: Effects of Depression Comorbidity and Treatment
}

\author{
Hee-Joon Lee', Ju-Wan Kim¹, Hee-Ju Kang', Sung-Wan Kim¹, II-Seon Shin', Young-Joon Hong'2, \\ Young-Keun Ahn' ${ }^{2}$, Myung-Ho Jeong ${ }^{2}$, Jin-Sang Yoon ${ }^{1}$, and Jae-Min Kim ${ }^{1} \bowtie$ \\ ${ }^{1}$ Department of Psychiatry, Chonnam National University Medical School, Gwangju, Republic of Korea \\ 'Department of Cardiology, Chonnam National University Medical School, Gwangju, Republic of Korea
}

\begin{abstract}
Objective The role of obsessive-compulsive symptoms (OCS) in patients with acute coronary syndrome (ACS) is not well elucidated. This study investigated the association between OCS and the long-term prognosis of ACS in tandem with depression comorbidity and treatment.

Methods A cross-sectional baseline study and a nested 24-week double-blind escitalopram-placebo controlled trial were carried out between May 2007 and March 2013, and then a 5-12-year follow-up for major adverse cardiac events (MACE) was conducted. A total of 1,152 patients with ACS were stratified by baseline depression comorbidity and treatment allocation into four groups: no depression (706 patients), depression and taking escitalopram (149 patients), depression and taking a placebo (151 patients), and depression and receiving medical care as usual (CAU; 146 patients). OCS were evaluated using the Symptom Checklist-90-Revised Obsessive-Compulsive symptom domain. During the follow-up, Kaplan-Meier event rates for MACE outcomes were calculated, and hazard ratios were estimated using Cox regression models after adjusting for a range of covariates.

Results A higher OCS score at baseline was associated with a worse ACS prognosis after adjusting for relevant covariates and across MACE outcomes. This association varied according to the depression comorbidity. The association was significant in patients without depression and depressive patients receiving placebos and CAU, but not in depressive patients on escitalopram.

Conclusion Evaluating OCS and depression is recommended during the early phase of ACS. Treatment for OCS may improve the longterm cardiac outcomes of patients with ACS.

Psychiatry Investig 2019;16(11):843-851
\end{abstract}

Key Words Acute coronary syndrome, Obsessive-compulsive disorder, Depression, Longitudinal studies, Treatment outcome.

\section{INTRODUCTION}

Acute coronary syndrome (ACS) is a life-threatening disease and a significant disease burden associated with high morbidity and mortality. ${ }^{1} \mathrm{~A}$ risk-factor evaluation is important for ACS patients, and several psychosocial variables have been considered prognostic predictors of ACS. Depression has been most extensively investigated, but anxiety, social isolation, and stress have also received research. ${ }^{2}$

Received: September 30, 2019 Accepted: October 22, 2019

$\triangle$ Correspondence: Jae Min Kim, MD, PhD

Department of Psychiatry, Chonnam National University Medical School, 160 Baekseo-ro, Dong-gu, Gwangju 61469, Republic of Korea

Tel: +82-62-220-6143, Fax: +82-62-225-2351, E-mail: jmkim@chonnam.ac.kr

(c) This is an Open Access article distributed under the terms of the Creative Commons Attribution Non-Commercial License (https://creativecommons.org/licenses/by$\mathrm{nc} / 4.0$ ) which permits unrestricted non-commercial use, distribution, and reproduction in any medium, provided the original work is properly cited.
Of the psychosocial factors, obsessive-compulsive symptoms (OCS) or disorder (OCD) belonged to the "anxiety disorder" category before the introduction of the Diagnostic and Statistical Manual of Mental Disorders-Fifth Edition (DSM5). ${ }^{3}$ Therefore, most previous studies on ACS have focused on anxiety rather than on OCS separately. Moreover, studies on the role of OCS on ACS prognosis are scarce and have presented inconclusive findings. Two cross-sectional studies reported that OCS are more common in patients with ACS than those without. ${ }^{4,5} \mathrm{~A}$ cohort study evaluated anxiety disorder as an ACS prognostic factor and reported that OCD was not associated with readmission and/or cardiac mortality at 12 months after the ACS event, but was significantly associated with them at 5 years. However, fewer than 20 OCD patients were observed. ${ }^{6,7}$ No other studies have evaluated the direct association between OCS and the ACS prognosis. Previous studies 
have many limitations, such as a small sample size, short follow-up period, or methodological problems.

Depression may be an important factor that impacts the association between OCS and cardiac outcomes in patients with ACS, as depression is a common comorbidity of OCD. ${ }^{8} \mathrm{~A}$ comorbid depressive feature in patients with OCD is accepted as a poor-prognosis factor, ${ }^{9}$ and antidepressants, particularly selective serotonin reuptake inhibitors (SSRIs), are effective for treating both OCS and depression. ${ }^{10}$ However, no study has considered depression comorbidity and treatment to investigate this issue. To resolve these unanswered questions, we investigated the effects of OCS on the long-term cardiac outcomes of ACS, as well as further evaluated this association according to the status of depression comorbidity and antidepressant treatment.

\section{METHODS}

\section{Study overview and participants}

The present analyses were performed using data from a larger naturalistic study of patients with ACS - the Korean DEPression in ACS (K-DEPACS) study, which also included a nested randomized clinical trial for patients with depression and ACS - the Escitalopram for DEPression in ACS (EsDEPACS) study. The original design and main findings of the K-DEPACS and EsDEPACS studies have been published, ${ }^{11,12}$ and eligibility criteria are described in the online Supplementary Material. Written informed consent was collected for both studies, which were approved by the Chonnam National University Hospital Institutional Review Board (IRB No. 06-050).
The outline and participant-recruitment process for the present analysis are presented in Figure 1.

\section{K-DEPACS baseline evaluations}

Participants were recruited from patients who were hospitalized due to ACS $(n=4,809)$ at the Department of Cardiology, Chonnam National University Hospital, Gwangju, South Korea from 2006 to 2012. This department was nominated by the Korean Circulation Society to serve as the central coordinating center for the Korea Acute Myocardial Infarction Registry (KAMIR) because of the large number of ACS patients seen here and the high quality of data acquisition and management here. ${ }^{13}$ Patients were treated by the study cardiologists based on international guidelines for managing ACS. ${ }^{14}$ Eligible participants $(n=1,152)$ were examined for baseline evaluations as inpatients within 2 weeks post-ACS, with a mean of $6.3 \pm 2.4$ [standard deviation (SD)] days. OCS were evaluated using the Symptom Checklist-90-Revised (SCL90-R). ${ }^{15}$ This is a self-administered 90 -item questionnaire consisting of multiple-choice questions with a 5-point (1-5) scale of distress, ranging from "not-at-all" to "extremely distressed." The Obsessive-Compulsive symptom dimension of the SCL-90-R consists of 10 of the total 90 items. The SCL$90-\mathrm{R}$ is used widely in clinical situations and in research because of its appropriate design for use in individuals with medical conditions ${ }^{15}$ including cardiac disorders. ${ }^{16,17}$ Major or minor depressive disorder was diagnosed by psychiatrists using the Mini-International Neuropsychiatric Interview, a structured diagnostic psychiatric interview for disorders in the DSM-IV. ${ }^{18}$ According to these criteria, patients are diagnosed

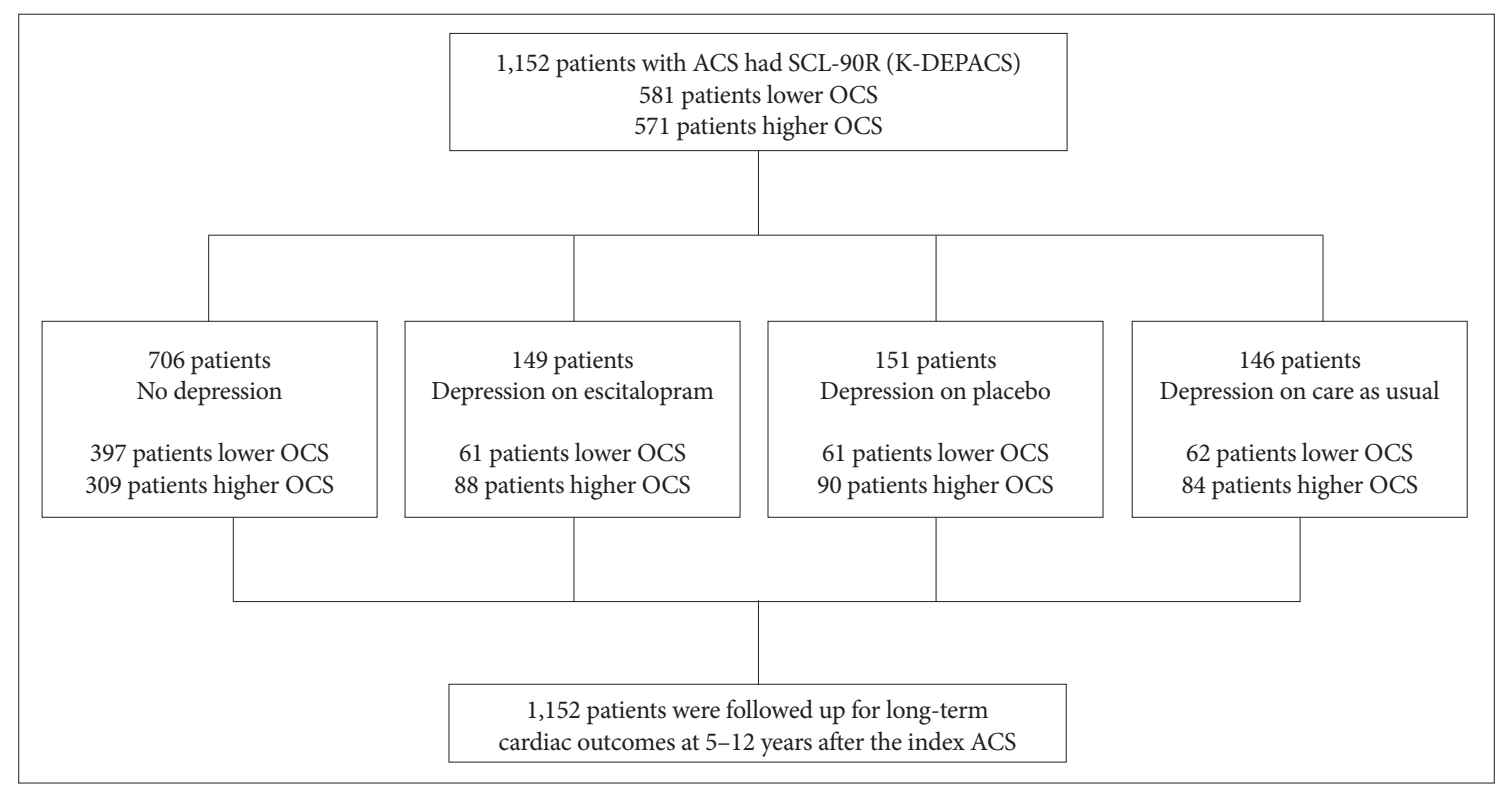

Figure 1. Study outline and participant allotment. ACS: acute coronary syndrome, K-DEPACS: Korean DEPression in ACS, OCS: obsessivecompulsive symptoms, SCL-90-R: Symptom Checklist-90-Revised. 
with major depressive disorder if they have at least one core symptom (i.e., depressed mood or loss of interest) and at least four other symptoms of depression. A diagnosis of minor depressive disorder is made if patients have at least one core symptom and at least two others, but fewer than five symptoms in total.

Baseline characteristics that could potentially affect cardiac outcomes were collected..$^{19}$ Demographic data on age, gender, education, marital status, living alone, accommodation, and employment status were obtained. The self-completed Beck Depression Inventory (BDI), ${ }^{20}$ and previous and family histories of depression were recorded to evaluate depressive symptoms. The following cardiovascular risk factors were ascertained: diagnosed hypertension and diabetes mellitus, hypercholesterolemia by fasting serum total cholesterol level (>200 mg/dL), obesity by the body mass index, reported current smoking status, and previous and family histories of ACS. Cardiac severity status was identified according to ACS diagnosis [myocardial infarction (MI) or unstable angina], Killip classification, ${ }^{21}$ left ventricular ejection fraction, and serum levels of troponin I and creatine kinase-MB.

\section{The nested EsDEPACS study}

Of the 446 individuals with a baseline diagnosis of a depressive disorder, 300 agreed to be randomized into the EsDEPACS study (ClinicalTrial.gov registry number: NCT00419471), a 24-week, double-blind, escitalopram $(n=149)$ or placebo $(n=$ 151) controlled trial. The first patient was enrolled in May 2007 and the last patient completed the follow-up evaluation in March 2013. Flexible doses of escitalopram (5, 10, 15, or 20 $\mathrm{mg}$ ) or a matched placebo were administered and determined according to the investigators' clinical decision, considering the patients' response and tolerance. Mean doses at the last visit were $7.6 \pm 3.7 \mathrm{mg}$ for the escitalopram group and $8.5 \pm 3.9$ $\mathrm{mg}$ for the placebo group. Examinations were scheduled at baseline, and at weeks $4,8,12,16,20$, and 24 thereafter. As previously reported, the main results of this trial demonstrated the superiority of the escitalopram treatment for alleviating depressive symptoms. ${ }^{11}$ The remaining 146 patients who declined participation $(n=123)$ or were ineligible $(n=23 ; 12$ for taking disallowed drugs, 6 with a history of neuropsychiatric illness, and 5 for participating in other drug trials) for participation in the trial received medical care as usual (CAU) for ACS. Higher participation rates were noted in those with more severe depression (i.e., major vs. minor depressive disorder). Of patients receiving escitalopram and a placebo, 57.0\% (85/ $149)$ and $55.6 \%(84 / 151)$ were diagnosed with a major depressive disorder, respectively, compared to $22.6 \%$ (33/146) of patients who received CAU.

\section{Long-term cardiac outcomes}

All participants were approached for follow-up evaluations of cardiac outcomes in 2017, 5-12 years after the index ACS, or until they died. The median follow-up duration was 8.4 years, and the mean was $8.7 \pm 1.5$ years. Comprehensive evaluations for cardiac outcomes were possible for this study because KAMIR manages and records detailed electronic data on hospital admissions, deaths, recurrent MI, and percutaneous coronary intervention (PCI). The primary endpoint was a major adverse cardiac event (MACE), which was a composite of all-cause mortality, MI, and PCI. Secondary endpoints were all-cause mortality, cardiac death (defined as sudden death for which no other explanation was available; death from arrhythmia, or after MI or heart failure; or death caused by heart surgery or endocarditis), MI, and PCI. An independent endpoint committee composed of study cardiologists adjudicated all potential events and was blinded to the participants' psychiatric comorbidities and the treatment randomization status.

\section{Statistical analyses}

As there have been no validated cutoffs published to categorize the severity of OCS, scores on the Obsessive-Compulsive symptom dimension of the SCL-90-R were dichotomized at the median value into less- and more-severe-OCS groups. Although this classification was arbitrary, it has been used in previous studies. ${ }^{22}$ Baseline demographic and clinical characteristics were compared between the less- and more-severeOCS groups using t-tests or $\chi^{2}$ tests, as appropriate. Characteristics significantly associated with OCS $(\mathrm{p}<0.05)$ and other variables with potential effects on MACE were used as covariates in further adjusted analyses. ${ }^{19}$ Kaplan-Meier models were used to compare the cumulative proportion of participants experiencing a composite and individual MACE (defined according to the date of the first event for each patient) between those with less- or more-severe OCS. Cox proportional hazards models were used to compare time to the first composite and individual MACE after adjusting for the potential covariates described above between the two groups. According to the depression comorbidity and treatment status at baseline, the patients were divided into four groups: no depression $(n=706)$, depression and taking escitalopram $(n=149)$, depression and taking a placebo $(n=151)$, and depression and receiving CAU ( $n=146)$. To evaluate the effects of depression comorbidity and treatment status on the associations between OCS and a composite and individual MACE, the same Cox proportional hazards models were used for these four groups separately, and the interaction terms were calculated after adjusting for all potential covariates. Additional sensitivity analyses were conducted using severity of OCS as a continuous exposure variable to re-examine its effect beyond the 
binary categorical approach. All statistical tests were two-sided with a significance level of 0.05 . Statistical analyses were carried out using SPSS software (ver. 21.0; IBM Corporation, Armonk, NY, USA).

\section{RESULTS}

\section{Baseline demographic and clinical characteristics by severity of OCS}

Median and mean values of the OCS dimension based on the SCL-90-R were 13.0 (interquartile range, 11.0-15.0) and $13.8 \pm 4.1$, respectively. The baseline characteristics between patients with less- and more-severe OCS are compared in Table 1 . More-severe OCS were significantly associated with a higher BDI score and a history of depression. These character- istics were included as covariates in subsequent analyses. In addition, age, education, all cardiac risk factors, and current cardiac status variables were also considered covariates because they have been associated with cardiac outcomes in previous studies. ${ }^{19,23}$

\section{Effects of OCS on the occurrence of a MACE}

The primary endpoint (composite MACE) occurred in 446 (38.7\%) participants. Considering secondary endpoints, allcause mortality occurred in 211 (18.3\%), cardiac death in 111 (9.6\%), MI in 110 (9.5\%), and PCI in 162 (14.1\%) participants. Figure 2 illustrates the cumulative risk of a composite MACE in subjects with less- and more-severe OCS. A significant difference was observed-composite MACE incidences were $31.3 \%(182 / 581)$ and $46.2 \%(264 / 571)$ in the less- and more-

Table 1. Baseline characteristics of patients with OCS 2 weeks after being diagnosed with ACS

\begin{tabular}{|c|c|c|c|c|}
\hline & Lower OCS $(\mathrm{N}=581)$ & Higher OCS (N=571) & Statistical coefficient & $\mathrm{p}$ \\
\hline \multicolumn{5}{|c|}{ Socio-demographic characteristics, N (\%) } \\
\hline Age, mean (SD) years & $58.8(11.2)$ & $58.4(11.5)$ & $\mathrm{t}=0.541$ & 0.589 \\
\hline Gender, female & $162(27.9)$ & $166(29.1)$ & $\chi^{2}=0.200$ & 0.655 \\
\hline Education, mean $(\mathrm{SD})$ years & $9.9(4.8)$ & $9.6(4.6)$ & $\mathrm{t}=0.903$ & 0.367 \\
\hline Unmarried marital status & $88(15.1)$ & $88(15.4)$ & $\chi^{2}=0.016$ & 0.900 \\
\hline Living alone & $60(10.3)$ & $52(9.1)$ & $\chi^{2}=0.489$ & 0.485 \\
\hline Housing, rented & $77(13.3)$ & $92(16.1)$ & $\chi^{2}=1.881$ & 0.170 \\
\hline Currently unemployed & $216(37.2)$ & $234(41.0)$ & $\chi^{2}=1.750$ & 0.186 \\
\hline \multicolumn{5}{|l|}{ Depression characteristics, N (\%) } \\
\hline BDI, mean (SD) score & $8.0(8.1)$ & $11.7(8.6)$ & $\mathrm{t}=-7.494$ & $<0.001$ \\
\hline Previous depression & $14(2.4)$ & $28(4.9)$ & $\chi^{2}=5.099$ & 0.024 \\
\hline Family history of depression & $14(2.4)$ & $19(3.3)$ & $\chi^{2}=0.872$ & 0.350 \\
\hline \multicolumn{5}{|l|}{ Cardiac risk factors, $\mathrm{N}(\%)$} \\
\hline Hypertension & $291(50.1)$ & $265(46.4)$ & $\chi^{2}=1.559$ & 0.212 \\
\hline Diabetes mellitus & $130(22.4)$ & $105(18.4)$ & $\chi^{2}=2.818$ & 0.093 \\
\hline Hypercholesterolemia & $281(48.4)$ & $291(51.0)$ & $\chi^{2}=0.778$ & 0.378 \\
\hline Obesity & $245(42.2)$ & $245(42.9)$ & $\chi^{2}=0.064$ & 0.800 \\
\hline Current smoker & $199(34.3)$ & $218(38.2)$ & $\chi^{2}=1.923$ & 0.165 \\
\hline Previous ACS & $159(27.4)$ & $164(28.7)$ & $\chi^{2}=0.262$ & 0.609 \\
\hline Family history of ACS & $19(3.3)$ & $20(3.5)$ & $\chi^{2}=0.048$ & 0.827 \\
\hline \multicolumn{5}{|l|}{ Current cardiac status } \\
\hline \multicolumn{5}{|l|}{ ACS diagnosis, N (\%) } \\
\hline Myocardial infarction & $422(72.6)$ & $407(71.3)$ & $\chi^{2}=0.262$ & 0.609 \\
\hline Unstable angina & $159(27.4)$ & $164(28.7)$ & & \\
\hline Killip class > 1, N (\%) & $98(16.9)$ & $108(18.9)$ & $\chi^{2}=0.822$ & 0.365 \\
\hline LVEF, mean (SD) \% & $61.0(11.7)$ & $61.4(10.9)$ & $t=-0.640$ & 0.522 \\
\hline Troponin I, mean (SD) mg/dL & $9.7(15.1)$ & $9.3(13.8)$ & $\mathrm{t}=0.406$ & 0.685 \\
\hline CK-MB, mean (SD) mg/dL & $17.9(40.3)$ & $16.3(33.2)$ & $\mathrm{t}=0.734$ & 0.463 \\
\hline
\end{tabular}

Statistical coefficients were calculated using t-tests or $\chi^{2}$ tests, as appropriate. OCS: obsessive-compulsive symptoms, ACS: acute coronary syndrome, BDI: Beck Depression Inventory, LVEF: left ventricular ejection fraction, CK-MB: creatine kinase-MB 
severe-OCS groups (long-rank $\mathrm{p}<0.001$ ), respectively. Figure 3 illustrates the cumulative risk of individual MACE components in the two groups. Significant differences were observed in the incidences of all-cause mortality $(16.0 \%$ and $20.7 \%$ in the less- and more-severe-OCS groups, respectively; log-rank $\mathrm{p}=0.035)$, MI (6.9\% and $12.3 \%$ in the less- and more-severe-

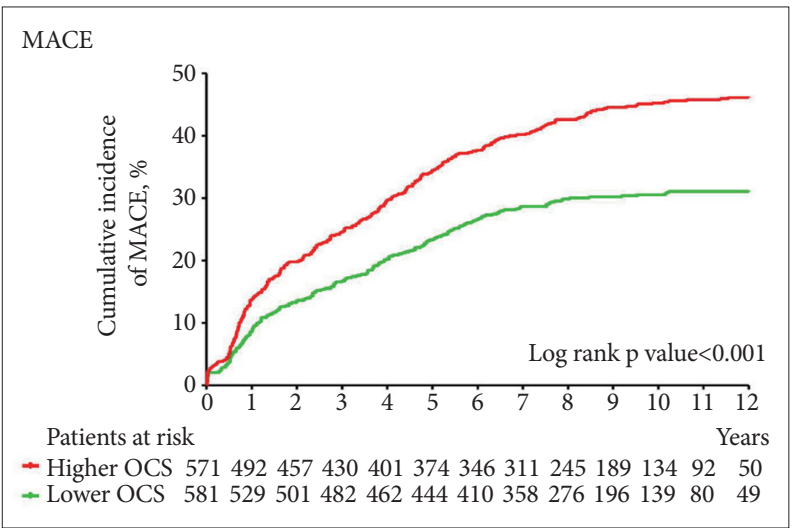

Figure 2. Cumulative incidence of composite MACEs. MACE: major adverse cardiac events, OCS: obsessive-compulsive symptoms.
OCS groups, respectively; log-rank $\mathrm{p}=0.001)$, and PCI $(10.7 \%$ and $17.5 \%$ in the less- and more-severe-OCS groups, respectively; log-rank $\mathrm{p}=0.001$ ), but not in the incidence of cardiac death. The effects of more-severe OCS at baseline on MACE outcomes after adjustment are summarized as hazards ratios with 95\% confidence intervals in parentheses in the "all participants" rows of Table 2 . The strength of the associations decreased, but remained significant for composite MACE, allcause mortality, MI, and PCI outcomes after adjusting for age, education, BDI score, history of depression, hypertension, diabetes, dyslipidemia, obesity, smoking, previous and family history of ACS, ACS diagnosis, Killip class, left ventricular ejection fraction, and serum levels of troponin I and creatine kinase-MB at baseline.

\section{Effects according to depression comorbidity and treatment status}

The effects of OCS on the incidences of MACE according to depression comorbidity and treatment status are summarized in Table 2. More-severe OCS at baseline in participants without depression were significantly associated with a com-
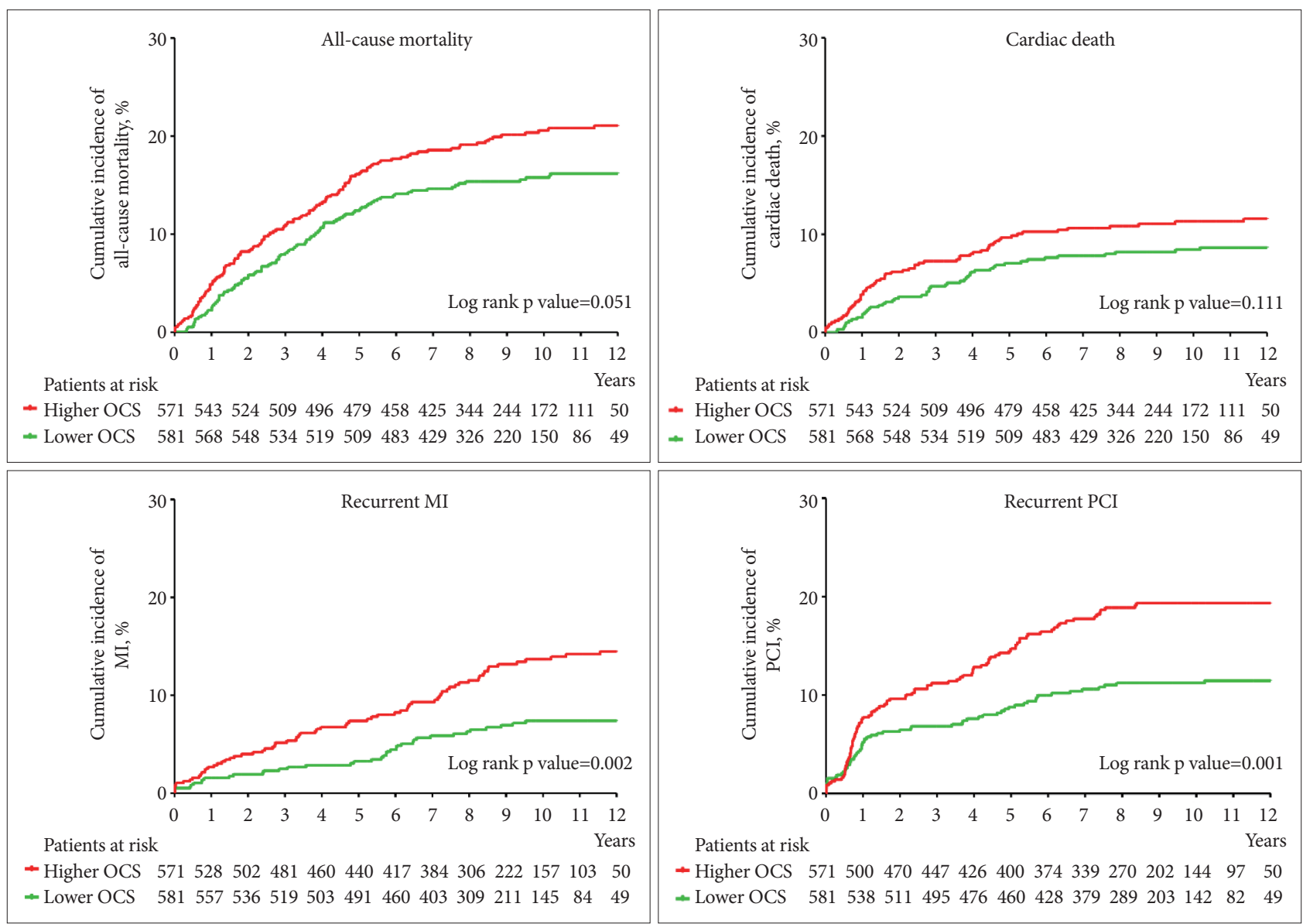

Figure 3. Cumulative incidence of the individual major adverse cardiac event components of allcause mortality, cardiac death, MI, and PCl. MI: myocardial infarction, PCl: percutaneous coronary intervention, OCS: obsessive-compulsive symptoms. 
Table 2. Effects of OCS on MACEs in 1,152 patients with ACS

\begin{tabular}{|c|c|c|c|c|c|}
\hline \multirow[t]{2}{*}{ Events } & \multirow{2}{*}{$\begin{array}{l}\text { Group by depression } \\
\text { comorbidity and treatment status }\end{array}$} & \multicolumn{2}{|c|}{$\begin{array}{l}\text { Kaplan-Meier event rate, } \\
\mathrm{N}(\%)\end{array}$} & \multicolumn{2}{|c|}{$\begin{array}{c}\text { Hazards ratios } \\
\text { (95\% confidence intervals) }\end{array}$} \\
\hline & & Lower OCS & Higher OCS & Unadjusted & Adjusted§ \\
\hline \multirow[t]{5}{*}{ MACE } & All participants & $182(31.3)$ & $264(46.2)$ & $1.63(1.35-1.96)^{\ddagger}$ & $1.60(1.32-1.94)^{\ddagger}$ \\
\hline & No depression & $105(26.4)$ & $112(36.2)$ & $1.45(1.11-1.90)^{\dagger}$ & $1.50(1.13-1.98)^{\dagger}$ \\
\hline & Depression on escitalopram & $24(39.3)$ & $37(42.0)$ & $1.08(0.65-1.80)$ & $1.25(0.71-2.22)$ \\
\hline & Depression on placebo & $24(39.3)$ & $57(63.3)$ & $1.86(1.15-2.99)^{*}$ & $1.68(1.02-2.77)^{*}$ \\
\hline & Depression on care as usual & $29(46.8)$ & $58(69.0)$ & $1.83(1.17-2.87)^{\dagger}$ & $2.14(1.31-3.50)^{\dagger}$ \\
\hline All-cause & All participants & $93(16.0)$ & $118(20.7)$ & $1.34(1.02-1.76)^{*}$ & $1.34(1.02-1.79)^{*}$ \\
\hline \multirow[t]{4}{*}{ Mortality } & No depression & $48(12.1)$ & $52(16.8)$ & $1.44(0.98-2.14)$ & $1.47(0.97-2.22)$ \\
\hline & Depression on escitalopram & $13(21.3)$ & $18(20.5)$ & $0.96(0.47-1.96)$ & $1.27(0.57-2.83)$ \\
\hline & Depression on placebo & $14(23.0)$ & $23(25.6)$ & $1.15(0.59-2.12)$ & $1.23(0.59-2.55)$ \\
\hline & Depression on care as usual & $18(29.0)$ & $25(29.8)$ & $1.05(0.57-1.92)$ & $1.06(0.51-2.18)$ \\
\hline \multirow[t]{5}{*}{ Cardiac death } & All participants & $48(8.3)$ & $63(11.0)$ & $1.38(0.95-1.96)$ & $1.37(0.93-2.03)$ \\
\hline & No depression & $27(6.8)$ & $23(7.4)$ & $1.13(0.65-1.97)$ & $1.18(0.66-2.13)$ \\
\hline & Depression on escitalopram & $6(9.8)$ & $10(11.4)$ & $1.15(0.42-3.16)$ & $2.03(0.54-7.57)$ \\
\hline & Depression on placebo & $6(9.8)$ & $14(15.6)$ & $1.61(0.62-4.18)$ & $1.73(0.61-4.91)$ \\
\hline & Depression on care as usual & $9(14.5)$ & $16(19.0)$ & $1.35(0.59-3.05)$ & $1.81(0.58-5.64)$ \\
\hline \multirow[t]{5}{*}{ Myocardial infarction } & All participants & $40(6.9)$ & $70(12.3)$ & $1.92(1.30-2.83)^{\dagger}$ & $1.74(1.17-2.59)^{\dagger}$ \\
\hline & No depression & $26(6.5)$ & $25(8.1)$ & $1.30(0.75-2.25)$ & $1.31(0.74-2.33)$ \\
\hline & Depression on escitalopram & $6(9.8)$ & $7(8.0)$ & $0.79(0.27-2.36)$ & $2.82(0.46-17.08)$ \\
\hline & Depression on placebo & $3(4.9)$ & $20(22.2)$ & $5.23(1.55-17.62)^{\dagger}$ & $5.00(1.40-17.88)^{*}$ \\
\hline & Depression on care as usual & $5(8.1)$ & $18(21.4)$ & $2.94(1.09-7.94)^{*}$ & $3.50(1.19-10.30)^{*}$ \\
\hline \multirow{5}{*}{$\begin{array}{l}\text { Percutaneous coronary } \\
\text { intervention }\end{array}$} & All participants & $62(10.7)$ & $100(17.5)$ & $1.76(1.28-2.41)^{\ddagger}$ & $1.78(1.29-2.47)^{\dagger}$ \\
\hline & No depression & $41(10.3)$ & $38(12.3)$ & $1.24(0.80-1.93)$ & $1.40(0.88-2.23)$ \\
\hline & Depression on escitalopram & $6(9.8)$ & $13(14.8)$ & $1.52(0.58-4.01)$ & $1.65(0.57-4.79)$ \\
\hline & Depression on placebo & $7(11.5)$ & $23(25.6)$ & $2.37(1.02-5.52)^{*}$ & $2.43(0.94-6.31)$ \\
\hline & Depression on care as usual & $8(12.9)$ & $26(31.0)$ & $2.85(1.29-6.30)^{*}$ & $3.78(1.57-9.11)^{\dagger}$ \\
\hline
\end{tabular}

${ }^{*} \mathrm{p}<0.05,{ }^{\dagger} \mathrm{p}<0.01,{ }^{\ddagger} \mathrm{p}<0.001$, §adjusted for age, education, Beck Depression Inventory score, history of depression, hypertension, diabetes, dyslipidemia, obesity, smoking, previous and family history of ACS, ACS diagnosis, Killip class, left ventricular ejection fraction, and serum levels of troponin I and creatine kinase-MB at baseline. OCS: obsessive-compulsive symptoms, MACEs: major adverse cardiac events, ACS: acute coronary syndrome

posite MACE but were not associated with any individual MACE outcome. These significant associations were not found with any MACE outcome in depressed patients taking escitalopam. Significant associations were detected with composite MACE and MI outcomes in depressed patients taking a placebo. In depressive patients receiving CAU, there were significant associations with a composite MACE, and MI and PCI outcomes. All of these associations were similar before and after adjustment. The results of additional sensitivity analyses using the scores based on the Obsessive-Compulsive symptom dimension of the SCL-90-R as continuous variables are summarized in Supplementary Table 1 (in the online-only Data Supplement). The strengths of the associations did not change substantially but the $\mathrm{p}$-values were slightly higher compared to those from the analysis using the scale as a binary variable.

\section{DISCUSSION}

The main findings were that more-severe OCS at the early phase of ACS was associated with worse long-term cardiac outcomes, but this association varied according to the depression comorbidity and treatment status-it was significant in patients without depression and in depressed patients taking a placebo or receiving CAU, but not in depressed patients taking escitalopram. These findings were robust after adjusting for the relevant covariates and across MACE outcomes.

Several mechanisms are plausible for the observed associations between more-severe OCS and worse prognosis of ACS. 
First, patients with OCD have relatively low serum adiponectin and high serum resistin levels compared to those of normal controls. ${ }^{24,25}$ Adiponectin and resistin are related to metabolic disturbances, including cholesterol levels and glucose tolerance. ${ }^{25}$ Therefore, ACS patients with more-severe OCS are more liable to have metabolic syndrome in a long-term follow-up. Similarly, OCD patients in the general population have higher rates of atherosclerosis than those without OCD. ${ }^{26}$ Moreover, OCD is associated with an increased risk of metabolic and cardiovascular complications after the mean 22year follow-up. ${ }^{27}$ In the present study, cardiac risk factors, including diabetes mellitus and hypercholesterolemia, did not differ between the less- and more-severe-OCS groups at baseline (Table 1). However, OCS may have contributed to metabolic disturbances over the 5-12-year follow-up period, although follow-up examinations were not carried out. Second, patients with OCD have persistent anxiety and therefore have an exhausted autonomic nervous system. ${ }^{28}$ In a functional brain magnetic resonance imaging study, activities increased in the limbic and paralimbic areas, indicating that the dorsolateral prefrontal cortex was hyperactivated in OCD patients. ${ }^{29}$ A meta-analysis reported that OCD results in less total sleep time and a higher amount of awake time than in people without OCD. This result is similar to how obstructive sleep apneahypopnea affects the autonomic nervous system by hypo-oxygenation, which is a well-known risk factor for ACS. ${ }^{30}$ Third, individuals with OCD have a core cognitive deficit called "low tolerance for uncertainty," which gives rise to compulsive behavior. ${ }^{31}$ Therefore, ACS patients with more-severe OCS are likely to have concerns about uncertainty that would worsen their heart problems. A controlled study using a gambling task revealed that OCD patients exhibited neuropsychiatric deficits. It is suspected that compulsions represent immediate rewards; thus, OCD patients are less sensitive to the future consequences of their choices. ${ }^{32}$ This psychological impairment in OCD patients seems to make them adhere less frequently to medical treatment, which leads to a recurrence of ACS. Moritz reported lower scores for social and emotional quality of life (QOL) variables, as physical QOL in OCD is compromised relative to healthy subjects both before and after biopsychosocial OCD treatment. $^{33}$

Notably, OCS did not affect ACS prognosis as much when patients received escitalopram treatment. This finding could be explained several ways. First, the antidepressive effect of escitalopram should be considered. We have reported that escitalopram was effective for treating depression in patients with ACS and improved long-term cardiac outcomes. ${ }^{11}$ In this study, OCS were significantly correlated with depressive symptoms evaluated using the BDI (Table 1); therefore, OCS may have improved along with the improvement in depres- sion. However, the associations between OCS and cardiac outcomes remained significant after adjusting for depressive symptoms. Second, escitalopram is also used for treating OCD and OCS. ${ }^{34}$ Therefore, the anti-OCS effect of escitalopram itself may have mitigated the negative consequences. Similarly, a Swedish nationwide cohort study reported that patients with OCD taking SSRIs had significantly lower risks of metabolic and cardiovascular complications compared with those who were not taking an SSRI. ${ }^{27}$ High SSRI doses are needed conventionally to treat OCD. ${ }^{34}$ Although lower doses (mean, 7.6 $\mathrm{mg}$ ) were used in the present study, OCS rather than OCD were evaluated. Third, escitalopram may normalize metabolic and autonomic disturbances, which have adverse effects on cardiac outcomes. ${ }^{35,36}$

Several issues should be considered before drawing conclusions. First, OCS rather than the diagnosis of OCD were evaluated and treated as an exposure variable. OCS are less formal and less accurate than OCD for categorizing the disease state, thus this categorization may have led to overestimates of the strength of the association. However, the numbers of OCD patients in previous studies were very low; ${ }^{6,7}$ therefore, it would be difficult to draw conclusions due to limited statistical power. In addition, OCS may reflect general distress more than OCD per se. ${ }^{37}$ In the present study, the association was confirmed using the score on the ObsessiveCompulsive symptom dimension of the SCL-90-R as a binary and continuous variable. Second, OCS were evaluated using the Obsessive-Compulsive symptom dimension of the SCL90-R. The Yale-Brown Obsessive-Compulsive Scale (YBOCS) is the gold-standard method for evaluating OCS. ${ }^{38}$ However, there are studies showing that the Obsessive-Compulsive symptom dimension of the SCL-90-R has strong internal consisten$\mathrm{cy},{ }^{15,37}$ and a moderate to high correlation with the YBOCS. ${ }^{39}$ Moreover, the SCL-90-R is a useful and convenient tool to screen psychiatric disease in various settings, including in physical disorder clinics worldwide. ${ }^{40}$ Third, recruitment was carried out at a single site, which may limit the generalizability of the present findings. However, a single center study has potential strengths in terms of consistency in evaluation and treatment. Other considerations are that no data were available on patients who refused to participate in the recruitment process of the K-DEPACS baseline participants; therefore, the representativeness of the sample cannot be confirmed. Additionally, the CAU group was not randomly assigned but was formed by patients with depression who refused or were ineligible to take part in the clinical trial; and no attempt was made to investigate the effect of treatment (or a placebo) for depression on OCS during the follow-up period.

Nevertheless, our study had several strengths. The followup for cardiac outcomes was comprehensive, long, and com- 
plete. The study design was unique in that a prospective observational study and a randomized placebo controlled interventional study were conducted. Participants were recruited at baseline consecutively from all eligible patients with recent ACS, which increased sample homogeneity. All measurements, including those based on the SCL-90-R for psychiatric and cardiovascular characteristics, were well validated. A range of covariates was considered in the analyses.

In summary, OCS had adverse effects on ACS prognosis, but possible beneficial modifying effects of treatment were found. These findings have several important implications. From a clinical perspective, evaluations of OCS along with depression are recommended for patients who recently developed ACS. The SCL-90-R questionnaire is simple and easy for ACS patients to complete even at the early hospitalization stage. This procedure could make it more likely for further appropriate treatment to improve long-term cardiac outcomes. Studies about other antidepressants or non-pharmacological psychiatric treatments, such as cognitive behavioral therapy for the more-severe-OCS group, are recommended to confirm the present findings. Additional time and medical costs may be involved besides the usual cardiologic practice; therefore, future studies need to investigate both the efficacy and cost-effectiveness of any intervention.

\section{Supplementary Materials}

The online-only Data Supplement is available with this article at https://doi.org/10.30773/pi.2019.0259.

\section{Acknowledgments}

This study was funded by a National Research Foundation of Korea grant (NRF-2015M3C7A1028899) and was supported by the Basic Science Research Program through the National Research Foundation of Korea funded by the Ministry of Science, ICT and Future Planning (grant NRF-2016R 1A2A2A05919518).

\section{Conflicts of Interest}

The authors have no potential conflicts of interest to disclose.

\section{Author Contributions}

Conceptualization: Jae-Min Kim, Jin-Sang Yoon, Young-Keun Ahn. Data curation: Lee-Hee Joon, Hee-Joo Kang. Formal analysis: Sung-Wan Kim, Hee-Joon Lee, Young-Joon Hong. Funding acquisition: Il-Seon Shin. Investigation: Lee-Hee Joon, Ju-Wan Kim, Jae-Min Kim. Methodology: Hee-Joo Kang, Myung-Ho Jeong. Project administration: Jae-Min Kim. Resources: Young-Joon Hong, Myung-Ho Jeong. Software: Hee-Joo Kang, Young-Keun Ahn. Supervision: Jin-Sang Yoon. Validation: Ju-Wan Kim. Visualization: Hee-Joon Lee. Writing_original draft: Hee-Joon Lee, Jae-Min Kim. Writing_-review \& editing: Hee-Joon Lee, Jin-Sang Yoon, Jae-Min Kim.

\section{ORCID iDs}

Jae Min Kim https://orcid.org/0000-0001-7409-6306

Hee-Joon Lee https://orcid.org/0000-0001-7409-6306
https://orcid.org/0000-0001-9611-8238

\section{REFERENCES}

1. Johansson S, Rosengren A, Young K, Jennings E. Mortality and morbidity trends after the first year in survivors of acute myocardial infarction: a systematic review. BMC Cardiovasc Disord 2017;7;17:53.

2. Kuper $H$, Marmot M, Hemingway H. Systematic review of prospective cohort studies of psychosocial factors in the etiology and prognosis of coronary heart disease. Semin Vasc Med 2002;2:267-314.

3. American Psychiatric Association. Diagnostic and Statistical Manual of Mental Disorders. 5th ed. Washington D.C.: American Psychiatric Association; 2013.

4. Bezgin $\mathrm{CH}$, Bezgin T, Kesebir S. Temperament and character profiles and psychiatric comorbidities in patients with coronary artery or valvular heart disease: relationship with cardiac disease severity. J Clin Med Res 2016;8:202-209.

5. Hamideh pour OJ, Iraj S, Bahman AK. Compare the obsessive-compulsive disorders between coronary heart disease and healthy subjects. J Nov Appi Sci 2014;3:283-286.

6. Parker G, Owen C, Brotchie H, Hyett M. The impact of differing anxiety disorders on outcome following an acute coronary syndrome: time to start worrying? Depress Anxiety 2010;23:302-309.

7. Parker G, Hyett M, Hadzi-Pavlovic D, Brotchie H, Walsh W. GAD is good? Generalized anxiety disorder predicts a superior five-year outcome following an acute coronary syndrome. Psychiatry Res 2011;188: 383-389.

8. Ruscio AM, Stein DJ, Chiu WT, Kessler RC. The epidemiology of obsessive-compulsive disorder in the National Comorbidity Survey Replication. Mol Psychiatry 2010;15:53-63.

9. Overbeek T, Schruers K, Vermetten E, Griez E. Comorbidity of obsessive-compulsive disorder and depression: prevalence, symptom severity, and treatment effect. J Clin Psychiatry 2002;63:1106-1112.

10. Spies M, Knudsen GM, Lanzenberger R, Kasper S. The serotonin transporter in psychiatric disorders: insights from PET imaging. Lancet Psychiatry 2015;2:743-755.

11. Kim JM, Bae KY, Stewart R, Jung BO, Kang HJ, Kim SW, et al. Escitalopram treatment for depressive disorder following acute coronary syndrome: a 24-week double-blind, placebo-controlled trial. J Clin Psychiatry 2015;76:62-68.

12. Kim JM, Stewart R, Lee YS, Lee HJ, Kim MC, Kim JW, et al. Effect of escitalopram vs placebo treatment for depression on long-term cardiac outcomes in patients with acute coronary syndrome: a randomized clinical trial. JAMA 2018;24;320:350-358.

13. Lee KH, Jeong MH, Kim HM, Ahn Y, Kim JH, Chae SC, et al. Benefit of early statin therapy in patients with acute myocardial infarction who have extremely low low-density lipoprotein cholesterol. J Am Coll Cardiol 2011;11;58:1664-1671.

14. Anderson JL, Adams CD, Antman EM, Bridges CR, Califf RM, Casey DE Jr, et al. 2012 ACCF/AHA focused update incorporated into the ACCF/AHA 2007 guidelines for the management of patients with unstable angina/non-ST-elevation myocardial infarction: a report of the American College of Cardiology Foundation/American Heart Association Task Force on Practice Guidelines. Circulation 2013;11;127:e663e828.

15. Derogatis LR. SCL-90-R: Administration, Scoring \& Procedures Manual-II for the Revised Version and Other Instruments of the Psychopathology Rating Scale Series. Towson, MD: Clinical Psychometric Research; 1992.

16. Follick MJ, Gorkin L, Smith TW, Capone RJ, Visco J, Stablein D. Quality of life post-myocardial infarction: effects of a transtelephonic coronary intervention system. Health Psychol 1988;7:169-182.

17. Allison TG, Williams DE, Miller TD, Patten CA, Bailey KR, Squires RW, et al. Medical and economic costs of psychologic distress in patients with coronary artery disease. Mayo Clin Proc 1995;70:734-742.

18. Sheehan DV, Lecrubier Y, Sheehan KH, Amorim P, Janavs J, Weiller E, et al. The Mini-International Neuropsychiatric Interview (M.I.N.I.): the 
development and validation of a structured diagnostic psychiatric interview for DSM-IV and ICD-10. J Clin Psychiatry 1998;59(Suppl 20):2233; quiz 34-57.

19. Jaffe AS, Krumholz HM, Catellier DJ, Freedland KE, Bittner V, Blumenthal JA, et al. Prediction of medical morbidity and mortality after acute myocardial infarction in patients at increased psychosocial risk in the Enhancing Recovery in Coronary Heart Disease Patients (ENRICHD) study. Am Heart J 2006;152:126-135.

20. Beck AT, Ward CH, Mendelson M, Mock J, Erbaugh J. An inventory for measuring depression. Arch Gen Psychiatry 1961;4:561-571.

21. Killip T 3rd, Kimball JT. Treatment of myocardial infarction in a coronary care unit. A two year experience with 250 patients. Am J Cardiol 1967;20:457-464.

22. Núñez-Rodríguez MH, Miranda Sivelo A. Psychological factors in gastroesophageal reflux disease measured by scl-90-R questionnaire. Dig Dis Sci 2008;53:3071-3075.

23. Panteghini M. Role and importance of biochemical markers in clinical cardiology. Eur Heart J 2004;25:1187-1196.

24. Atmaca M, Ustundag B, Metin K, Topuz M. Low plasma adiponectin levels in obsessive-compulsive disorder. J Affect Disord 2009;117:205207.

25. Ari M, Ozturk OH, Bez Y, Arica S, Can Y, Erduran D. Serum adiponectin and resistin levels in patients with obsessive compulsive disorder. J Affect Disord 2012;136:979-982.

26. Paterniti S, Zureik M, Ducimetière P, Touboul PJ, Fève JM, Alpérovitch A. Sustained anxiety and 4-year progression of carotid atherosclerosis. Arterioscler Thromb Vasc Biol 2001;21:136-141.

27. Isomura K, Brander G, Chang Z, Kuja-Halkola R, Rück C, Hellner C, et al. Metabolic and cardiovascular complications in obsessive-compulsive disorder: a total population, sibling comparison study with long-term follow-up. Biol Psychiatry 2018;1;84:324-331.

28. Pittig A, Arch JJ, Lam CW, Craske MG. Heart rate and heart rate variability in panic, social anxiety, obsessive-compulsive, and generalized anxiety disorders at baseline and in response to relaxation and hyperventilation. Int J Psychophysiol 2013;87:19-27.

29. Simon D, Kaufmann C, Kniesche R, Kischkel E, Kathmann N. Autonomic responses and neural-cardiac coupling during individually tailored symptom provocation in obsessive-compulsive disorder. J Anxi- ety Disord 2013;27:635-644.

30. Díaz-Román A, Perestelo-Pérez L, Buela-Casal G. Sleep in obsessivecompulsive disorder: a systematic review and meta-analysis. Sleep Med 2015;16:1049-1055.

31. Hezel DM, McNally RJ. A theoretical review of cognitive biases and deficits in obsessive-compulsive disorder. Biol Psychol 2016;121:221232.

32. Cavedini P, Riboldi G, D’Annucci A, Belotti P, Cisima M, Bellodi L. Decision-making heterogeneity in obsessive-compulsive disorder: ventromedial prefrontal cortex function predicts different treatment outcomes. Neuropsychologia 2002;40:205-211.

33. Moritz S, Rufer M, Fricke S, Karow A, Morfeld M, Jelinek L, et al. Quality of life in obsessive-compulsive disorder before and after treatment. Compr Psychiatry 2005;46:453-459.

34. Bloch MH, McGuire J, Landeros-Weisenberger A, Leckman JF, Pittenger C. Meta-analysis of the dose-response relationship of SSRI in obsessivecompulsive disorder. Mol Psychiatry 2010;15:850-855.

35. Dawood T, Barton DA, Lambert EA, Eikelis N, Lambert GW. Examining endothelial function and platelet reactivity in patients with depression before and after SSRI therapy. Front Psychiatry 2016;18;7:18.

36. Alvares GA, Quintana DS, Hickie IB, Guastella AJ. Autonomic nervous system dysfunction in psychiatric disorders and the impact of psychotropic medications: a systematic review and meta-analysis. J Psychiatry Neurosci 2016;41:89-104.

37. Woody SR, Steketee G, Chambless DL. Reliability and validity of the Yale-Brown Obsessive-Compulsive Scale. Behav Res Ther 1995;33:597605.

38. Goodman WK, Price LH, Rasmussen SA, Mazure C, Fleischmann RL, Hill CL, et al. The Yale-Brown Obsessive Compulsive Scale. I. Development, use, and reliability. Arch Gen Psychiatry 1989;46:1006-1011.

39. Grabill K, Merlo L, Duke D, Harford KL, Keeley ML, Geffken GR, et al. Assessment of obsessive-compulsive disorder: a review. J Anxiety Disord 2008;22:1-17.

40. Schmitz N, Kruse J, Heckrath C, Alberti L, Tress W. Diagnosing mental disorders in primary care: the General Health Questionnaire (GHQ) and the Symptom Check List (SCL-90-R) as screening instruments. Soc Psychiatry Psychiatr Epidemiol 1999;34:360-366. 


\section{SUPPLEMENTARY MATERIALS}

\section{Eligibility Criteria for the K-DEPACS and EsDEPACS participants}

For the K-DEPACS study entry, inclusion criteria were as follows: 1) aged 18-85 years; 2) confirmed ACS by investigation (the presence of ST-segment elevation MI was determined by $>30$ min of continuous chest pain, a new ST-segment elevation $\geq 2$ mm on at least two contiguous electrocardiographic leads, and creatine kinase-MB more than three times normal; the presence of non-ST-segment elevation MI was diagnosed by chest pain and a positive cardiac biochemical marker without new ST-segment elevation; and the presence of unstable angina was determined by chest pain within the preceding $72 \mathrm{~h}$ with or without ST-T wave changes or positive cardiac biochemical markers); 3) ability to complete study questionnaires; 4) ability to understand the study objectives and sign informed consent. Exclusion criteria were: 1) occurrence of ACS while hospitalized for another reason; 2) ACS developing less than 3 months after a coronary artery bypass graft procedure; 3 ) uncontrolled hypertension (systolic blood pressure (BP) $>180 \mathrm{~mm} \mathrm{Hg}$ or diastolic $\mathrm{BP}>100 \mathrm{~mm} \mathrm{Hg}$ ); 4) resting heart rate $<40 / \mathrm{min}$; 5) severe physical illnesses threatening life or interfering with the recovery from ACS; 6) persistent clinically significant laboratory abnormalities in complete blood cell counts, thyroid tests, renal function tests, and liver function tests. For the EsDEPACS study entry, additional inclusion criteria were as follows: 1) BDI>10;2) major or minor depressive disorder according to DSM-IV criteria. Additional exclusion criteria were: 1) concomitant use of class I antiarrhythmic medications, reserpine, guanethidine, clonidine, methyldopa, lithium, anticonvulsants, antipsychotics, or antidepressants; 2) history of neuropsychiatric illnesses such as dementia, Parkinson's disease, brain tumor, psychosis, bipolar disorder, alcoholism, or other substance dependence; 3) pregnancy; 4) participating in other drug trials. 
Supplementary Table 1. Effects of obsessive-compulsive symptoms (one point increase in the Obsessive-Compulsive symptom dimension of Symptom Checklkist-90-Revised) on major adverse cardiac outcome (MACE) in 1152 patients with acute coronary syndrome (ACS)

\begin{tabular}{|c|c|c|c|}
\hline \multirow{2}{*}{ Events } & \multirow{2}{*}{$\begin{array}{c}\text { Group by depression comorbidity } \\
\text { and treatment status }\end{array}$} & \multicolumn{2}{|c|}{ Hazards ratios ( $95 \%$ confidence intervals) } \\
\hline & & Unadjusted & Adjusted§ \\
\hline \multirow[t]{5}{*}{ MACE } & All participants & $1.08(1.07-1.09)^{\ddagger}$ & $1.07(1.06-1.08)^{\ddagger}$ \\
\hline & No depression & $1.06(1.05-1.07)^{\dagger}$ & $1.06(1.05-1.08)^{\dagger}$ \\
\hline & Depression on escitalopram & $1.00(0.98-1.02)$ & $1.01(0.09-1.03)$ \\
\hline & Depression on placebo & $1.03(1.01-1.05)^{*}$ & $1.01(1.00-1.03)^{*}$ \\
\hline & Depression on care as usual & $1.09(1.07-1.11)^{\dagger}$ & $1.10(1.08-1.12)^{\dagger}$ \\
\hline All-cause & All participants & $1.03(1.02-1.04) \dagger$ & $1.03(1.01-1.04)^{*}$ \\
\hline \multirow[t]{4}{*}{ Mortality } & No depression & $1.02(0.99-1.04)$ & $1.01(0.99-1.05)$ \\
\hline & Depression on escitalopram & $1.00(0.98-1.02)$ & $1.00(0.98-1.02)$ \\
\hline & Depression on placebo & $1.00(0.98-1.02)$ & $1.01(0.99-1.04)$ \\
\hline & Depression on care as usual & $1.03(0.99-1.08)$ & $1.03(0.99-1.08)$ \\
\hline \multirow[t]{5}{*}{ Cardiac death } & All participants & $1.01(0.99-1.03)$ & $1.01(0.99-1.04)$ \\
\hline & No depression & $1.00(0.98-1.05)$ & $1.00(0.98-1.06)$ \\
\hline & Depression on escitalopram & $1.00(0.97-1.04)$ & $1.01(0.99-1.06)$ \\
\hline & Depression on placebo & $1.02(0.99-1.05)$ & $1.02(0.98-1.07)$ \\
\hline & Depression on care as usual & $1.01(0.98-1.05)$ & $1.01(0.98-1.06)$ \\
\hline \multirow[t]{5}{*}{ Myocardial infarction } & All participants & $1.06(1.05-1.07)^{\dagger}$ & $1.05(1.04-1.08)^{\dagger}$ \\
\hline & No depression & $1.02(0.98-1.05)$ & $1.02(0.98-1.06)$ \\
\hline & Depression on escitalopram & $0.99(0.96-1.06)$ & $1.00(0.96-1.08)$ \\
\hline & Depression on placebo & $1.09(1.05-1.22)^{\dagger}$ & $1.08(1.04-1.23)^{\dagger}$ \\
\hline & Depression on care as usual & $1.04(1.01-1.14)^{*}$ & $1.05(1.02-1.16)^{*}$ \\
\hline \multirow[t]{5}{*}{ Percutaneous coronary intervention } & All participants & $1.07(1.06-1.08)^{\ddagger}$ & $1.07(1.06-1.09)^{\ddagger}$ \\
\hline & No depression & $1.01(0.98-1.03)$ & $1.01(0.98-1.05)$ \\
\hline & Depression on escitalopram & $1.02(0.98-1.08)$ & $1.02(0.98-1.10)$ \\
\hline & Depression on placebo & $1.07(1.02-1.22)^{*}$ & $1.08(1.02-1.23)^{*}$ \\
\hline & Depression on care as usual & $1.10(1.05-1.30)^{\dagger}$ & $1.11(1.06-1.35)^{\dagger}$ \\
\hline
\end{tabular}

${ }^{*} \mathrm{p}<0.05,{ }^{\dagger} \mathrm{p}<0.01,{ }^{\ddagger} \mathrm{p}<0.001$, §adjusted for age, education, Beck Depression Inventory score, history of depression, hypertension, diabetes, dyslipidemia, obesity, smoking, previous and family history of ACS, ACS diagnosis, Killip class, left ventricular ejection fraction, and serum levels of troponin I and creatine kinase-MB at baseline 